\title{
The Galactic Fountain
}

\author{
F.D. Kahn
}

Department of Physics and Astronomy, The University of Manchester, Manchester M13 9PL, England

\begin{abstract}
The Galactic fountain is driven by the hot inter-cloud medium (ICM). The ICM itself is heated by successive supernova explosions. This process introduces sufficient irregularities to prevent the fountain flow from being smooth. Instead it leads to the formation of a frothy medium above the disk (Avillez, Berry \& Kahn 1997). This paper describes the effect of successive supernova explosions, in a rather idealised case. The real case is still more complicated, but the present approach shows why it is so.
\end{abstract}

\section{Introduction}

Most of the Galactic interstellar matter is present in a disk, a relatively thin layer extending to some $100 \mathrm{pc}$ on either side of the Galactic plane. This material is largely contained in cool clouds where hydrogen, the dominant constituent, is present either in atomic $(\mathrm{H})$ or $\left(\mathrm{H}_{2}\right)$ molecular form. The atomic hydrogen or HI clouds have the kinematical properties of extreme Population I: they follow the rotation of the Galactic disk and have random speeds large enough to raise them to a maximum distance of $100 \mathrm{pc}$ or so from the plane. The typical temperature in an HI region may be taken to be $100 \mathrm{~K}$ and the smoothed-out density $2 \times 10^{-24} \mathrm{gm} \mathrm{cm}^{-3}$. The adjective smoothed-out is important here: the cool gas in reality fills only a few per cent of the available volume.

The inter-cloud medium (ICM) fills the space between the clouds. The ICM is hot, at typically $10^{6} \mathrm{~K}$, in contrast to the HI clouds. Its density is typically $10^{-26} \mathrm{gm} \mathrm{cm}^{-3}$ so that its pressure is typically $10^{-12}$ dyne $\mathrm{cm}^{-2}$. An important physical parameter is the sound speed in the ICM

$$
c_{s} \equiv\left(\frac{\gamma P_{\mathrm{o}}}{\rho_{h}}\right)^{1 / 2} \sim 1.3 \times 10^{7} \mathrm{~cm} \mathrm{~s}^{-1}
$$

an order of magnitude larger than the typical random speeds of extreme Population I objects. (In this formula $\mathrm{P}_{\mathrm{o}}$ and $\rho_{h}$ are respectively the pressure and the density in the ICM, and $\gamma$ is the ratio of specific heats.) The gravitational field of the Galaxy confines Population I objects to a disk with a half-thickness of $100 \mathrm{pc}$; the hot ICM should consequently rise to a height $c_{s}^{2} / g_{z}$ which is larger by two orders of magnitude. Here $g_{z}$ is the component of the Galactic gravitational field perpendicular to the disk; it has a value 
$10^{-8} \mathrm{~cm} \mathrm{~s}^{-2}$ at points well away from the Galactic plane. Clearly then the ICM should extend into a volume much thicker than that of the disk.

The dynamical relaxation time will be

$$
t_{\mathrm{dyn}}=\frac{c_{s}}{g_{z}}
$$

in any equilibrium structure attained by the ICM; for comparison, the rate of radiative heat loss is given by the cooling law

$$
\frac{D}{D t} \kappa^{3 / 2}=-q
$$

which is applicable in the range of temperatures $10^{5}<T<3 \times 10^{7} \mathrm{~K}$ (Kahn 1976). In this relation

$$
\kappa=P / \rho^{5 / 3}
$$

and $P$ and $\rho$ are respectively the pressure and the density of the ICM. The cooling rate is set by the parameter $q$ and itself depends on the composition of the gaseous medium concerned. For solar abundances, that is no depletion to dust grains, its value is $4 \times 10^{32} \mathrm{~cm}^{6} \mathrm{gm}^{-1} \mathrm{~s}^{-4}$. The resulting cooling time, due to radiative loss, is

$$
t_{\mathrm{cool}}=\frac{\kappa^{3 / 2}}{q}=\frac{P^{3 / 2}}{q \rho^{5 / 2}}
$$

and the hot ICM can reach a hydrostatic equilibrium only if

$$
t_{\text {cool }} \gg t_{\text {dyn }}
$$

or

$$
\frac{P}{\rho^{2}} \gg \frac{q}{g_{z}}
$$

With the present values of the physical parameters, the left hand side in this inequality is smaller, by a factor 4 , than the right hand side, with the result that the ICM can never attain equilibrium unless it is heavily depleted in metals. The hot gas consequently escapes from the thin Population I disk, but it eventually falls back because its thermal energy is too small for escape from the Galaxy.

In the most familiar models this flow is described as a fountain, or a fountain with some loss into a wind (Bregman 1980, Breitschwerdt, McKenzie \& Völk, 1993, Kahn 1994). The medium is generally treated as though it were smooth, having no structure on small scales. The upward flow either remains subsonic throughout (in Bregman's version) or starts subsonic and then passes through a critical level above which it is supersonic (in Kahn's version). The continuing radiative heat loss creates conditions which allow the transit through the sonic level. In all cases the gas is cold on its return flow. 
At first sight such models provide a very reasonable solution to the problem posed by the existence of a reservoir of hot gas in the Galactic disk with so much thermal energy that it cannot be held. The residence time of the ICM is only of order $Z_{o} / c_{h} \approx 10^{6}$ years, and here $Z_{o}$ is the half-thickness of the disk. Supernovae of type II continually heat more interstellar gas to replenish the ICM. According to the usual estimates, there are three such events per century in the Galaxy, or $3 \times 10^{4}$ every million years. The Galactic disk has an area of some $3 \times 10^{8} \mathrm{pc}^{2}$, so that there is one explosion per million years for every $10^{4} \mathrm{pc}^{2}$ of disk. In any period of a million years, that is within the characteristic escape time of the ICM gas, the typical distance between neighbouring explosions is therefore $100 \mathrm{pc}$, similar to the thickness of the disk.

Successive supernova explosions introduce a rather rugged structure into the interstellar medium. The outer parts of a SNR are shocked, compressed, and heated but not enough to stay hot for a million years. The gas on the outside cools off again, and then condenses into HI clouds with random speeds which are too small to smooth out the irregularities before the next explosion. The gas from the inner part of the SNR, by contrast, stays hot. If each remnant yields some $200 M_{\odot}$ of hot gas, then there is an adequate supply to compensate for the outflow to the fountain. But the question remains whether this flow can be smooth.

Recently Avillez et al. (1997) have tackled this problem in a computer simulation. Their results, presented in Figures 1 and 2 (see description in Avillez et al. (1997), show what the Galactic disk and halo can be expected to look like after evolving some hundreds of million years.

The simulation starts with the interstellar gas distributed in a smooth thin disk. Successive explosions cause the formation of a frothy disk, with typical half-thickness $1 \mathrm{kpc}$. Once disrupted by the explosions, the disk never returns to its initial state. Instead it seems to approach a state where there is cool gas present predominantly in a thin but irregular layer around the Galactic plane. The ICM is shown to extend into a thicker irregular layer where it is mixed with some of the cool gas, and where the mixture is in some sort of turbulent equilibrium. A smoother fountain flow appears to exist at greater heights. There seem also to be chimneys present (in Figure 2 (a) located at $x=200 \mathrm{pc}, z=-150 \mathrm{pc}$ ), where high pressure gas has forced its way out through relatively narrow channels (for a recent observation of a chimney, see Normandeau, Taylor \& Dewdney 1996.). The froth in the thick disk consists of bubbles of hot gas separated by thin regions of cold gas.

One great problem at present with such a calculation is that the computational grid is still rather coarse. The resolution is limited to $10 \mathrm{pc}$. It is therefore impossible to resolve clearly any cool sheets present. Typically such sheets are expected to be no more than one or two parsecs thick. Clearly there are important parts of the physics which the computation can only handle in an approximate way. Nevertheless the model developed by Avillez constitutes 
(a)

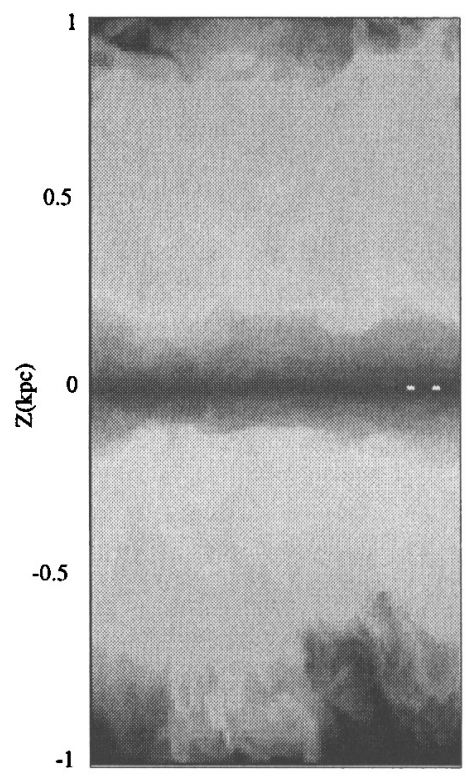

(c)

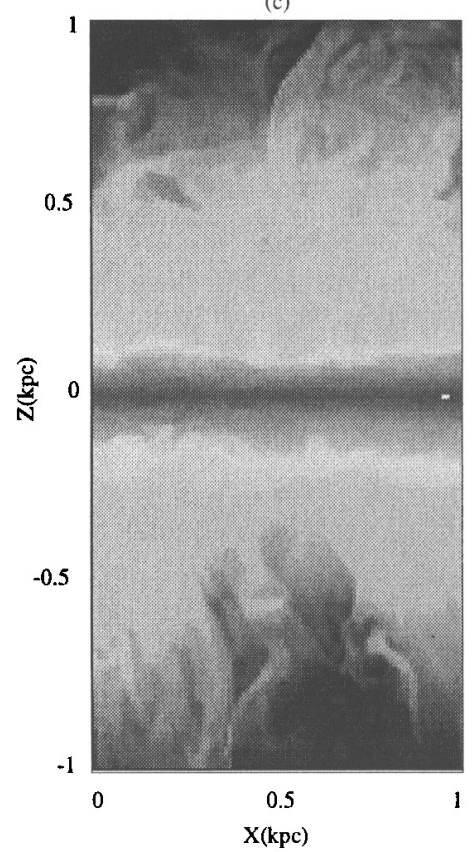

(b)

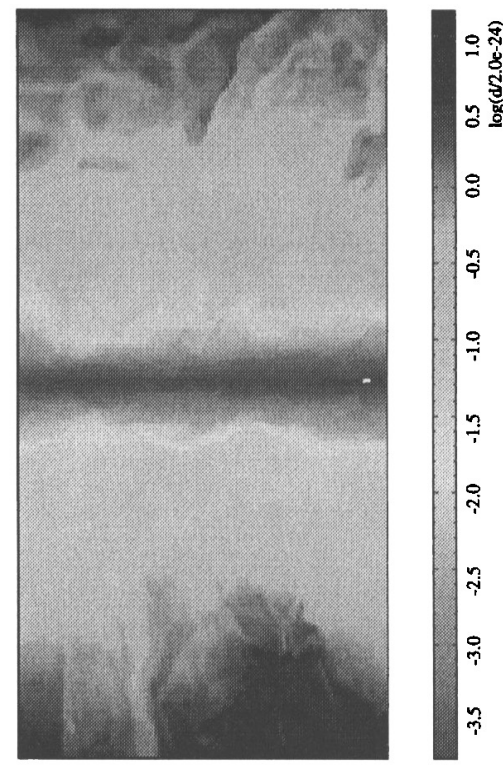

(d)

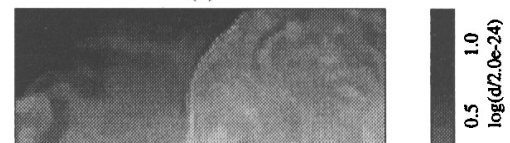

8

o

웅

$\frac{n}{3}$

ণั

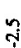

울

ก

Fig. 1. Density distribution in a vertical section perpendicular to the Galactic plane for times 280, 283, 286, and 289 Myr after start of calculations; (see Plate 5). 
(a)

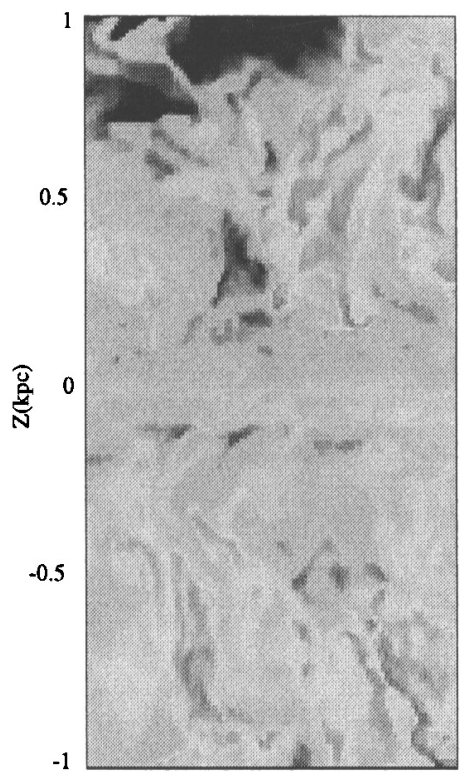

(c)

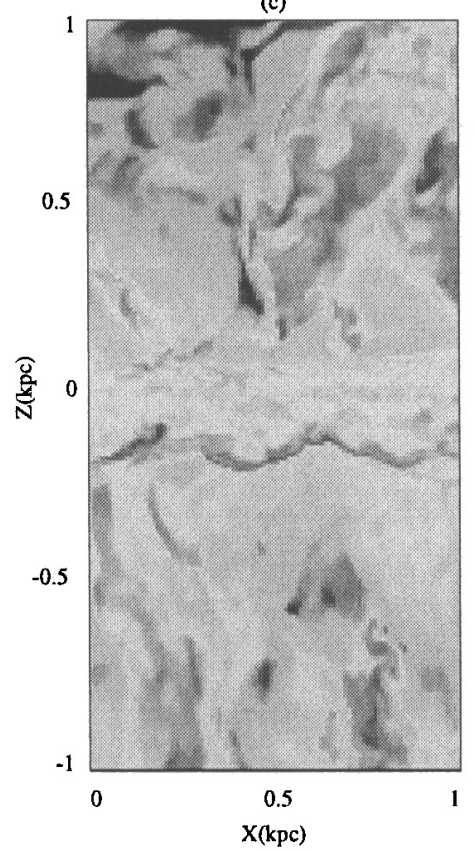

(b)

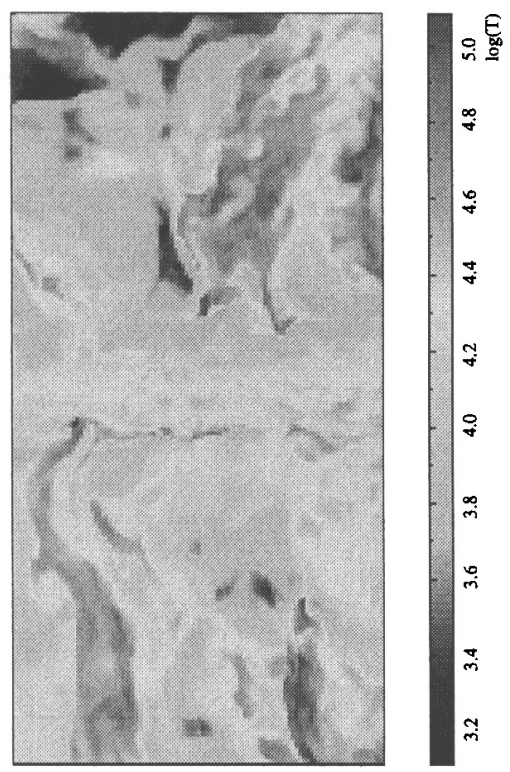

(d)

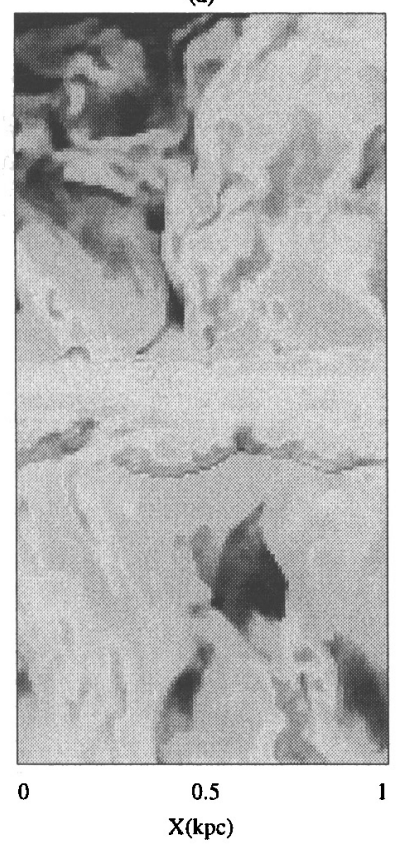

올

$\stackrel{\infty}{\forall}$

$\stackrel{\circ}{\circ}$

$\stackrel{+}{*}$

ษ

웅

冓

$\stackrel{\circ}{\circ}$

ti

m

Fig. 2. Temperature distribution in a vertical section perpendicular to the Galactic plane for times 280, 283, 286, and 289 Myr after start of calculations; (see Plate 6). 
an important step forward and agrees very much better with observation than the more primitive calculations, based on a smooth fountain flow. There may well be interesting magnetic effects in the flow, but the present calculation does not include them. Much better resolution would be needed for that in the computation.

\section{The Late Evolution of Supernova Remnants}

A supernova releases some $10^{53} \mathrm{erg}$ of energy, mostly in the form of neutrinos; a smaller amount $E$, typically $3 \times 10^{51} \mathrm{erg}$, is released in the form of kinetic energy of baryonic matter and destroys the parent star. It is important to discover how much of this energy is passed to the interstellar medium, and in what form. It is therefore worth while to follow the evolution of a remnant into its later stages.

The standard results are that the shock radius and the shock speed are, respectively,

$$
r=\left(\frac{2 E}{\rho_{\mathrm{o}}}\right)^{1 / 5} \tau^{2 / 5}
$$

and

$$
\dot{r}=\frac{2}{5}\left(\frac{2 E}{\rho_{\mathrm{o}}}\right)^{1 / 5} \tau^{-3 / 5}
$$

at time $\tau$ after the explosion; here $\rho_{o}$ is the density of the interstellar medium, assumed to be homogeneous. The adiabatic parameter $\kappa$ immediately postshock is given by

$$
\kappa_{s}^{3 / 2}=0.02 \frac{\dot{r}_{s}^{3}}{\rho_{\circ}}=0.002 \frac{E^{3 / 5}}{\rho_{\circ}^{8 / 5}} \tau^{-9 / 5}
$$

for comparison the mass of gas which has been shocked at that time is

$$
\mathcal{M}_{s}=\frac{4 \pi}{3} \rho_{\mathrm{o}}\left(\frac{2 E}{\rho_{\mathrm{o}}}\right)^{3 / 5} \tau^{6 / 5}
$$

so that there is a simple relation

$$
\kappa_{s} \mathcal{M}_{s}=0.10 \frac{E}{\rho_{\circ}^{2 / 3}}
$$

between the mass of gas engulfed by the shock, at any time, and the value of the adiabatic parameter immediately post-shock. The mass enclosed is conserved following any spherical shell, and so is the adiabatic parameter on the surface of that shell, as long as radiative heat loss has not yet become significant, that is during Phase II. It follows that relation (10) remains valid without the suffixes, until serious cooling sets in. 
A parcel of gas that was shocked at time $\tau$ will have cooled completely at time

$$
t=\tau+\frac{0.002 E^{3 / 5}}{q \rho_{\circ}^{8 / 5}} \tau^{-9 / 5}
$$

the first element of gas to do so must have passed through the shock at time

$$
\tau=\tau_{\star}=\frac{0.13 E^{3 / 14}}{q^{5 / 14} \rho_{\circ}^{4 / 7}}
$$

and will be completely cold when

$$
t=t_{I I}=\frac{0.20 E^{3 / 14}}{q^{5 / 14} \rho_{\circ}^{4 / 7}} ;
$$

this time marks the end of Phase II. Relation (6), which is still reasonably accurate then, shows that the radius of the SNR is

$$
r_{I I}=\frac{0.60 E^{2 / 7}}{q^{1 / 7} \rho_{\mathrm{o}}^{3 / 7}}
$$

at that time. With the parameters assumed here $r_{\mathrm{II}}=9.7 \times 10^{19} \mathrm{~cm}$ and $t_{\mathrm{II}}=1.7 \times 10^{12} \mathrm{~s}$. Much later, well into Phase III, only the gas for which

$$
\kappa_{s}^{3 / 2}>q t
$$

will still be hot; according to eq. (12) it amounts to a mass

$$
\mathcal{M}_{h}=\frac{0.10 E}{\rho^{2 / 3} q^{2 / 3}} t^{-2 / 3}
$$

At partial mass $\mathcal{M}$ within this distribution of hot gas, assumed to be spherically symmetrical, the adiabatic parameter is

$$
\kappa=\frac{0.10 E}{\rho_{\circ}^{2 / 3} \mathcal{M}}
$$

and the specific volume of the gas is

$$
v=\left(\frac{\kappa}{P}\right)^{3 / 5}=\frac{0.25 E^{3 / 5}}{\rho_{\mathrm{o}}^{2 / 5} \mathcal{M}^{3 / 5}} P^{-3 / 5}
$$

under pressure $P$. The volume occupied by the hot gas is therefore, after some algebra,

$$
V=\int_{0}^{\mathcal{M}_{h}} v d \mathcal{M}=\frac{0.25 E}{\rho_{\circ}^{2 / 3} P^{3 / 5} q^{4 / 15}} t^{-4 / 15}
$$

and so

$$
P=\frac{0.0092 E^{5 / 3}}{\rho_{\circ}^{10 / 9} q^{4 / 9}} r^{-5} t^{-4 / 9}
$$


for a spherical volume of radius $r$ at time $t$. It is convenient to introduce dimensionless radius $R$ and time $\theta$ by setting

$$
r=R r_{I I} \text { and } t=\theta t_{I I}
$$

and then

$$
P=p_{\star} R^{-5} \theta^{-4 / 9}
$$

where

$$
p_{\star}=0.24 E^{1 / 7} q^{3 / 7} \rho_{\circ}^{9 / 5}
$$

The pressure of the hot gas assists the expansion of the remnant shell even after the end of Phase II. The equation of motion

$$
\frac{\rho_{\circ}}{3} r \ddot{r}+\rho_{\circ} \dot{r}^{2}=P
$$

differs from the usual snowplough equation by the presence of the pressure term on the right. The equation makes no allowance for the mass of hot gas, and is really valid only long after time $t_{\mathrm{II}}$. It can be written

$$
\frac{1}{3} \frac{d}{d t} \rho_{\circ} r^{3} \dot{r}=\operatorname{Pr}^{2}
$$

in dimensionless variables and after some transformations becomes

$$
S^{3 / 4} \frac{d^{2} S}{d \theta^{2}}=\frac{12 p_{\star} t_{I I}^{2}}{\rho_{\mathrm{o}} r_{I I}^{2}} \theta^{-4 / 9}=0.32 \theta^{-4 / 9}
$$

with the definition

$$
S \equiv R^{4}
$$

The term on the right in eq. (28) represents the effect of the pressure of the hot gas on the dynamics of the shell. Without this term the equation describes the motion in the snowplough approximation, and then $S$ is linear in $\theta$. Clearly the snowplough treatment is valid at late times, when $\theta$ is large. At early times, during Phase II, the motion of the shell is described by equation (8), or in dimensionless form

$$
S=\theta^{8 / 5}
$$

The solution, for late times, which matches smoothly onto that for Phase II, at time $\theta=1$, is

$$
S=2.44 \theta\left(1-0.43 \theta^{-7 / 36}-0.16 \theta^{-1} \ldots\right)
$$

and so the dimensionless radius is

$$
R=1.25 \theta^{1 / 4}\left(1-0.11 \theta^{-7 / 36}-0.041 \theta^{-1}+\ldots\right)
$$

The dimensionless volume of the remnant is 


$$
\mathcal{V}=\frac{4 \pi}{3} R^{3}=8.18 \theta^{3 / 4}
$$

to adequate accuracy. Expressed in dimensionless terms the rate at which supernovae occur is

$$
N \equiv \nu r_{I I}^{3} t_{I I}
$$

in this formula

$$
\nu=(30 \text { years })^{-1}\left(6 \times 10^{10} \mathrm{pc}^{3}\right)^{-1}=6 \times 10^{-76} \mathrm{~cm}^{-3} \mathrm{~s}^{-1}
$$

is the rate in physical variables, and it follows that $N=0.00093$. A simple argument leads to an estimate for the length of time that passes before virtually the whole space in the Galactic disk must be filled by old SNR's. Suppose that to begin with the interstellar medium is homogeneous and that supernovae occur at rate $N$ from time $\Theta=0 \mathrm{on}$. A remnant started by an explosion at time $\Theta-\theta$ will have grown to volume $\mathcal{V}$ at time $\Theta$, and so the fractional volume occupied at time $\Theta$ is

$$
f=8.18 N \int_{0}^{\Theta} \theta^{3 / 4} d \theta=4.67 N \Theta^{7 / 4}
$$

The estimate is consequently that the remnants have filled all the available space when $f=1$ and $\Theta=0.41 N^{-4 / 7}$ so that $\Theta=22.1$, equivalent to a physical age $3.8 \times 10^{13} \mathrm{~s}$ or $1.2 \times 10^{6}$ years. The radius of the remnant will then be $R=2.17$, equivalent to $2.1 \times 10^{20} \mathrm{~cm}$ or $70 \mathrm{pc}$.

These estimates are simple but have the great drawback that they are inappropriate. The simulations described by Avillez, Berry \& Kahn (1997) show that the interstellar medium never returns to its initial state and that all supernova remnants evolve in an inhomogeneous medium.

Still, in the framework of the simple model, the thermal energy content of a remnant in Phase III is

$$
\mathcal{E}_{\text {ther }}=2 \pi P r^{3}=0.21 E \theta^{-17 / 18}
$$

and always small when compared with $E$. Typically $\mathcal{E}_{\text {ther }}=0.024 \mathrm{E}$ and $0.012 E$ at dimensionless times $\theta=10$ and 20 , respectively. With the data adopted here the total energy released, per unit time, in dynamical form by supernovae amounts to some $3 \times 10^{42} \mathrm{erg} \mathrm{s}^{-1}$ for the Galactic disk as a whole. Of this power about one or two per cent becomes available to drive the Galactic Fountain, enough for a mass loss rate of some $10^{-19} \mathrm{gm} \mathrm{cm}^{-2}$ $\mathrm{s}^{-1}$ with a speed of $100 \mathrm{~km} \mathrm{~s}^{-1}$ over an area of $6 \times 10^{8} \mathrm{pc}^{2}$. There seems to be satisfactory agreement. 


\section{A Supernova Exploding in a Low Density Region}

A second supernova may explode inside an existing SNR, an event much more likely to occur during Phase III than Phase II, and later rather than sooner. The dynamical energy of the second explosion goes almost entirely to the remaining hot gas. Before being shocked again this gas has a density distribution of the form

$$
\rho=\Omega r^{n}
$$

and $n=9 / 2$ in the present application. An expression for $\Omega$ is found from the condition that the mass of hot gas $\mathcal{M}_{h}$ (see relation (18)) is contained in a sphere of radius $1.25 r_{\mathrm{II}} \Theta^{1 / 4}$, so that, with some algebra,

$$
\Omega=0.148 \rho r_{I I}^{-9 / 2} \Theta^{-61 / 24}
$$

The effect of the second explosion can be found analytically, but only at the cost of some apparently drastic approximations. A similarity solution requires the second explosion to occur at the same place as the first, and the energy content of the remaining hot gas to be small compared with $E$. The second condition is easily met, but the first seems highly artificial. Nevertheless it makes little real difference if the second explosion takes place rather off centre, for the following reason. The shock from the second blast advances very fast through the interior, where the density is low, and slows down considerably later, as it runs into the higher density gas further out. An off-centre explosion creates a blast wave whose surface is elongated, to begin with, in the direction where the ambient density is lowest, but this period of asymmetrical expansion is only brief. The shock spends much more time moving through the bulk of the mass, which is near the boundary of the first remnant, and its surface becomes progressively more spherical.

Another difference is that a similarity solution strictly applies only when the explosion itself ejects a negligible mass. An actual supernova ejects a finite mass $M_{e j}$, say $5 M_{\odot}$; the mass of hot gas in the remnant can be shown to be

$$
\mathcal{M}_{h}=0.32 \mathcal{M}_{I I} \Theta^{-2 / 3}
$$

$\mathcal{M}_{\text {II }}$ being about $4000 M_{\odot}$ here. So if the second explosion takes place say 500,000 years after the first, when $\Theta \approx 9$, then the similarity solution holds if

$$
5 \ll 1280 f \times 9^{-2 / 3}
$$

Here $f$ is the fraction of the hot diffuse gas that has been overrun by the second shock; according to (41) it has to exceed about 2 per cent. In general the approximation holds.

The shock path is easily found from the virial theorem

$$
\frac{1}{2} \ddot{I}=2\left(E_{\text {ther }}+E_{\text {kin }}\right) \equiv 2 E
$$


where

$$
I \equiv \int_{0}^{\mathcal{M} *} r^{2} d \mathcal{M}
$$

Here define $\xi \equiv r / r_{s}$ and $\mu \equiv \mathcal{M} / \mathcal{M}_{s}$ where $r_{s}$ and $\mathcal{M}_{s}$ respectively are the shock radius and the mass that it encloses. Relation (42) holds provided no significant energy loss has occurred by radiation. The mass $\mathcal{M}_{\star}$ is fixed and larger than $\mathcal{M}_{s}$. The motion of the shock leads to a change $\Delta \mathrm{I}$ in the value of I because material within radius $r_{s}$ has been displaced outwards, and in the case of a general $n$

$$
\Delta I=\mathcal{M}_{s} r_{s}^{2} \int_{0}^{1} \xi^{2} d \mu-4 \pi \Omega \int_{0}^{r_{s}} r^{n+4} d r=\mathcal{M}_{s} r_{s}^{2}\left[\int_{0}^{1} \xi^{2} d \mu-\frac{n+3}{n+5}\right]
$$

The quantity $\mathcal{M}_{s} r_{s}^{2}$ varies like $t^{2}$ in a similarity solution; it follows that

$$
2 E=\frac{1}{2} \frac{d^{2} I}{d t^{2}}=\frac{\mathcal{M}_{s} r_{s}^{2}}{t^{2}}\left[\int_{0}^{1} \xi^{2} d \mu-\frac{n+3}{n+5}\right]=\frac{4 \pi \Omega r_{s}^{n+5}}{(n+3) t^{2}}\left[\int_{0}^{1} \xi^{2} d \mu-\frac{n+3}{n+5}\right]
$$

The strong shock condition leads to the boundary condition

$$
\frac{d \mu}{d \xi}=4(n+3)
$$

immediately behind the shock at $\xi=1$. The integral in (45) can then be well approximated by assuming that $\mu$ increases exponentially with $\xi$ as $\xi$ approaches unity and then (45) leads to

$$
2 E=\frac{4 \pi \Omega r_{s}^{n+5}}{(n+3) t^{2}}\left[\frac{2(n+3)}{2 n+7}-\frac{n+3}{n+5}\right]
$$

and so

$$
r_{s}^{n+5}=\frac{(n+5)(2 n+7)}{6 \pi} \frac{E t^{2}}{\Omega}
$$

and

$$
\dot{r}_{s}^{2}=\frac{2(2 n+7)}{3 \pi(n+5)} \frac{E}{\Omega} r_{s}^{-(n+3)}
$$

since

$$
\dot{r}_{S}=\frac{2 r}{(n+5) t}
$$

Here $n=9 / 2$ and so

$$
\dot{r}_{s}=0.36 \frac{E}{\Omega} r_{s}^{-15 / 2}
$$

The second shock reaches the edge of the original remnant when

$$
r_{s}=1.25 r_{I I} \Theta^{1 / 4}
$$

and then, from (38), (39) and (51), 


$$
\dot{r}_{s}^{2}=0.46 \frac{E}{\rho_{\circ} r_{I I}^{3}} \Theta^{2 / 3}=0.24\left(\frac{r_{I I}}{t_{I I}}\right)^{2} \Theta^{2 / 3}
$$

and the shock speed is $280 \Theta^{1 / 3} \mathrm{~km} \mathrm{~s}^{-1}$ there. The dynamical time, that is the time to reach the outer edge after the second explosion, is

$$
t_{\mathrm{dyn}}=\frac{4}{19} \frac{r_{s}}{\dot{r}_{s}}=0.43 t_{I I} \Theta^{-1 / 12}
$$

This time has to be compared with the cooling time $t_{\text {cool }}$ of the newly shocked gas. When the blast waves reaches the boundary of the old remnant

$$
t_{\text {cool }}=\frac{\kappa_{s}^{3 / 2}}{q}=\frac{0.02 \dot{r}_{s}^{3}}{q \Omega r_{s}^{9 / 2}}=0.78 t_{I I} \Theta^{29 / 12}
$$

and so

$$
\frac{t_{\text {cool }}}{t_{\mathrm{dyn}}}=1.82 \Theta^{5 / 2}
$$

with the shock speed found above, the post-shock temperature becomes

$$
T_{s}=10^{6} \Theta^{2 / 3} \mathrm{~K}
$$

and can be quite high if $\Theta$ is large enough, in other words if the second supernova is delayed sufficiently long.

\section{Conclusions}

The analytical approach can hardly be carried any further, since great complications will arise from the combined effect of many supernovae. The dynamical consequence of any one explosion is sensitively dependent on the state of the surrounding interstellar medium. If an explosion takes place in a region of low density then the surrounding gas is heated, but takes a long time to cool. The energy therefore becomes available to drive further motions. By contrast an explosion in a high density region is followed by (relatively) rapid heat loss, and much less dynamical effect. Any realistic calculation must strive to give an accurate description of the interstellar medium on quite small length scales.

\section{References}

Avillez M.A., Berry D.L. \& Kahn F.D., 1997, these proceedings.

Bregman J.N. 1980, ApJ 236, 577.

Breitschwerdt D., McKenzie J.F., Völk H.J., 1993, A\&A, 269, 54.

Kahn F.D., 1994, ApSS, 216, 325.

Normandeau M., Taylor A.R. \& Dewdney P.E., 1996, Nature 380, 687. 